\title{
SOME BASIC PRINCIPLES IN LEPROSY TREATMENT
}

\author{
E. MUIR
}

Up to the present day we have had little opportunity of studying Mycobacterium leprae outside the human body. It is true that we can make surmises from the analogies of other mycobacteria, such as those of rat leprosy or of the various forms of tuberculosis; but the differences between these latter and human leprosy are almost as striking as the resemblances, and too much must not be based upon such analogies.

\section{The Two Forms of Leprosy}

A vast difference exists between the more severe form of leprosy and the slight form which is localised and may often be abortive. What are the factors which create this difference? A mild epidemic of smallpox has been described alongside of a more severe one, and it was proved that this was due to two different strains of virus, the one more virulent than the other. But no one has seriously suggested that the severity of leprosy depends on the mycobacterial strain, and all available evidence is against such a supposition; the difference, whatever it is, lies in the host and not in the invading germ.

The main difference between the severe and the slight case of leprosy depends, at least in the first place, on the activity of tissue cells, especially those known variously as histiocytes, monocytes or macrophages. When the lepra bacillus enters the body its survival depends upon the behaviour of these cells and the degree to which they react locally to its presence, and there may be a wide difference in the degree and nature of this reaction.

There may be intense mytotic division so that the surrounding tissues become infiltrated with epithelioid cells and lymphocytelike cells packed together in dense nodules, with giant cells of the Langhan's type scattered here and there. In such a case the bacilli are either destroyed or are at least handicapped in their attempt to multiply.

On the other hand there may be only mild reaction; the cellular infiltration is at first slight and the bacilli instead of being destroyed multiply inside the cells and possibly also in the intercellular lymph spaces. Wandering cells may convey the bacilli to other sites. The bacilli multiplying inside the cytoplasm give rise to a vacuolated appearance characteristic of the well-known "lepra cell." 
In this latter condition not only do the bacilli survive and multiply, but they are able, as it were, to dig themselves in. The way in which that is done may best be described by quoting a translation of the description by Unna ${ }^{1}$.

"We know already a series of obstacles to medical results. The greatest of these is the torpid and very feeble reaction which the bacillus excites round about it in the tissues of the skin and nerves. It does not cause a violent inflammation, serous or purulent, but a simple hypertrophy of connective tissue, of its fibres and some of its cells, tending towards the encapsulation of the bacilli, although the epithelial cells of all kinds remain absolutely passive. In brief the bacillus insinuates itself into the tissue and is retained there, not expelled.

" A second obstacle is the obstruction by the bacilli of all the lymphatic spaces of the tissues in such a way that it is impossible for lively circulation to take place wherever a focus of lepra bacilli has established itself, and thus our remedies can only very slowly gain access. By this obstacle is explained the paradoxical fact that superficial lepromas of the skin are more difficult to heal than those deeper down in the subcutaneous tissue; for in the latter medicaments can penetrate better into the lymphatic system free from the skin.

"A third obstacle, entirely unique, is encountered in the bacillus itself, which produces in its interior a fatty substance; this is why it generally becomes more accessible to oily than to watery fluids. If it shares this difficulty with the tubercle bacillus, it surpasses it by far in local inaccessibility by surrounding itself with ' gloea' that is a mucoid vegetable mass consisting of dead and swollen bacilli, which partly consist of solid fatty substance. This gloea partly separates bacilli from living tissue and partly keeps the foci of bacilli from contact with our remedies.

"The inert vegetable mass, by which leprosy differs unfavourably from tuberculosis, is stained only with great difficulty, and up till now has always been mistaken for the protoplasm of animals cells".

We have described the two extremes of local reaction to the invading bacillus: the one, associated with high resistance and typical of neural leprosy, in which there is intense cellular reaction and in which the bacilli are entirely or almost entirely destroyed; and the other associated with low resistance and typical of lepromatous leprosy in which the cells act sluggishly and the bacilli multiply and dig themselves in. There are of course many stages and minor grades between the two.

\section{Two Objectives in Treatment}

In any well-established case of leprosy of the lepromatous type, treatment must aim at two objectives: (a) to strengthen and stimulate the cells to act more vigorously, and (b) to break down the triple defence of the bacilli. Care and restraint must be exercised, however, in bringing about the latter, and it must be carried out gradually and only in proportion as the cells have been stimulated and strengthened to deal effectively with the bacilli set free and brought into close contact with them. Otherwise the bacilli are likely to be carried to a distance and form fresh foci and thus extend the disease.

Keeping in mind these two objectives and the proviso that 
the bacillary triple defence must be broken down only with care, let us examine the various possible remedies and procedures for the treatment of leprosy.

(I) Stimulation and strengthening of the cells. In considering this we should be helped considerably if we knew the reasons underlying the immense difference referred to above between individual patients and the degree of their cellular reaction and resistance to invading bacilli. Is the degree of resistance hereditary? Is it dependent on the degree of infection, especially in early childhood? To what extent is it affected by the condition of endocrine glands such as the suprarenals or thyroid? The writer has seen a slight but long-standing single lesion become negative and disappear under thyroid treatment in a few weeks time.

Of one thing there can be no doubt, that the general health of the patient profoundly affects his resistance to the disease. Deterioration of health not only favours the spread of formerly stationary lesions, but it not infrequently results in tuberculoid lesions degenerating into those of the lepromatous type. On the contrary, it is seldom that a lepromatous case recovers (at least without serious deformity) unless he has continued for a considerable period in uniformly first class health.

The appearance in the neural type of leprosy of what is commonly known as the 'major tuberculoid lesion,' which especially in its more outstanding form is of the nature of a suddenly sensitized minor tuberculoid, is frequently followed by complete healing of the lesion or lesions which resolve and leave only scars. This apparently allergic phenomenon seems to correspond to extreme activation of the local cells, enabling them to destroy the comparatively few bacilli present.

The better known "lepra reaction" in lepromatous leprosy appears to be of a similar nature. The difference is that in place of a few bacilli in the reacting lesions there are myriads which it is beyond the power of the sensitized and activated cells to destroy. In consequence, though many bacilli may be destroyed, the disease tends to spread both locally through the lymph channels and to a distance through the blood. At the same time the toxæmia set up causes a general febrile reaction and tends to depress the general health of the patient still further.

The contrast between the reaction in tuberculoid and that in lepromatous lesions illustrates forcibly the fact already referred to-that breaking down of the triple defence of the bacilli in excess of the power of the cells to deal with the bacilli thus set free is dangerous and harmful. 
We know very little of the action of the most widely used remedy in leprosy-chaulmoogra on and its derivatives. 1 here is no reliable evidence that it acts directly on the bacillus. Does it activate the cells to destroy bacilli? when injected intradermally into skin lesions there is little doubt that it stimulates the cells locally; but what about its more generalised action? These are questions awaiting further investigation.

Local applications to the skin have the effect of stimulating the phagocytic cells and at the same time of freeing the bacilli and bringing them in contact with the cells. Unna in the article referred to above ${ }^{1}$ strongly advocates the vigorous use of various types of applications. He recommends iron baths, beginning at a temperature of $30^{\circ} \mathrm{C}$ and gradually heating; hot douches; friction with oil; ironing the skin with a hot iron, the skin being protected with a layer of flannel. These forms of treatment can be repeated day by day to different parts of the skin surface.

Various kinds of caustics can be used. One of the commonest is the application of a $I$ in 3 solution of trichloracetic acid. Carbon dioxide snow is particularly valuable in elevated nodules. One of the methods recommended by Unna was shaving off the outer parts of the skin, including nodules, with a razor after freezing with ethyl chloride. Cotton swabs soaked in a styptic are then applied and are fastened on with plaster. The next day the same part is again frozen and a caustic is applied. A small part of the nodular surface is thus dealt with daily. In ulcerating nodules, as distinct from trophic ulcers, a useful form of treatment is daily painting with $I$ in 3 trichloracetic acid till the leproma is absorbed and epithelium begins to grow at the edge.

Pressure is another form of local application useful in reducing at least the grosser forms of lesions. It can be applied by means of elastic bands, elastoplast or split bicycle tubes. Unna recommended applying pressure over a dressing of zinc oxide and glycerine.

As mentioned above, intradermal injection of hydnocarpus oil or its preparations forms a useful method of stimulating the local cells apart from any more general effect that the absorbed oil may have.

To get good results a combination or alternation of these forms of local treatment may be used, but passive applications should always be accompanied by active exercises. In the Mkambati Leprosy Institution in South Africa, where unusually good results are obtained, the patients spend $a$ considerable time every morning in bathing, inunction and various applications, and carefully regulated exercises; these are all done communally and 
under expert supervision. This is in addition to agricultural work in which they employ themselves for much of the rest of the day.

Active physical exercises have the effect of improving the metabolism and the general health. At the same time they can be used to prevent and remove the deformities of hands, feet and other parts of the body. Removal of dead bone in the feet, hygiene of the nose and the gums and teeth, care of early lesions of the eyes-all these are of the highest importance; failing them, the patient tends to become immobilized and his general resistance deteriorates in consequence.

None of the above recommendations are likely to be of much value unless they are backed by satisfactory nutrition. Not only must the diet be carefully balanced and regulated, but accompanying diseases which interfere with nutrition must be sought out and removed.

2. Breaking down bacillary defence. Both the passively received applications and the active exercises mentioned above, if carefully and skilfully regulated, have the effect of strengthening and stimulating the cells and of breaking down the triple defence mechanism of the bacilli so as to bring the latter in contact with the cells judiciously and only in such numbers as they are able to deal with effectively.

It should be mentioned here that we have a drug in our possession which has a specific effect in breaking down the defence mechanism of the bacilli-potassium iodide. This drug has been used from time to time, and as often abandoned on account of its dangerous qualities. In some lepromatous cases even a fraction of a grain has the effect of causing an acute generalised reaction with febrile symptoms, swelling up of leproma of skin, mucous membranes and nerves; though in most cases larger doses are necessary to produce such an effect. The liver, testicles, lymph nodes and any part of the body where masses of bacilli exist are liable to take part in the reaction. Iodide does not appear to have any effect on single bacilli, and large doses may be given without reaction in cases of the neural type with tuberculoid lesions in which, on careful examination, only single bacilli or occasional clumps of two or three are found with difficulty. In apparently neural cases, however, it may cause reaction in affected nerve trunks, presumably because they contain large clumps of bacilli. It would appear that iodide has the effect of dissolving or breaking up the mucoid gloea referred to above, which surrounds the bacilli and helps to isolate them from the surrounding living tissues: How it acts we do not know; but apparently it is not by 
direct action. For no reaction results from injection of iodide solution direct into leproma in a strength far in excess of the concentration that can be produced in the tissues by giving large doses by the mouth. Iodide can be used safely in the following two categories: (a) In lepromatous cases with first class physique in whom the bacilli have been reduced to such small numbers that repeated routine examinations of skin and mucosa fail to find them ${ }^{2,3}$. In such cases the administration of iodide in increasing, carefully regulated, doses breaks up the defence mechanism if the bacilli in residual foci show their presence by causing local nodules or macules, and enables the activated cells to destroy the broken up clumps; (b) In lepromatous cases with good general health, but with a fairly large infection as shown by microscopic examination use minute fractional doses beginning with one-tenth of a grain or less, to be repeated, it may be a week or a month later, only when all signs of reaction from the last dose (including rise in sedimentation rate) have passed away, and raised in amount only when the previous dose fails to produce any appreciable reaction. The essential is that bacilli should not be set free and brought in contact with the living tissues in greater numbers than the cells are able to destroy.

\section{Spontanleous Healing of I eipkomatous Cases}

Reference has been made above to relative cellular activity as determining the type of lesion, producing the tuberculoid if activity is high. the lepromatous if activity is low. It has been shown that the former will not infrequently heal up spontaneously, especially after a sudden "flare up " or reaction. There is another condition under which spontaneous resolution takes place. Very advanced lepromatous cases will occasionally heal up without special treatment, though almost always there are marked deformities left. This natural healing process may be speeded up by treatment.

Much has been written in recent years about treatment of leprosy with various dyes such as mercurochrome, methylene blue, trypan blue, fluorescine, \&c. The way in which these dyes act when given in repeated doses over a considerable period is illustrated by the following case of advanced lepromatous type and many years standing. The treatment consisted of fluorescine ro grains a day for seven months, methylene blue 4 grains a day for six months, and trypan blue 4 grains a day for over one month. These were given in the order mentioned, occasionally being discontinued for a few days. The lepromatous areas both cutaneous and subcutaneous showed intense staining and later 
began one or more at a time, to become inflamed and to suppurate. After discharging pus full of acid-fast bacilli each area in turn died down, leaving more or less marked scars. The same process occurred in the nose, mouth and throat. Frrom time to time there was severe gastro-intestinal disturbance which suggested that a somewhat similar occurrence was taking place in internal leproma. The patient began to cough up large quantities of pus apparently from the trachea. So severe was this condition on one occasion that his life had to be saved by an emergency tracheotomy; but after he had worn the tube for a few months it was possible to dispense with it. During this period the patient was having almost constant fever. One eye had been destroyed some years previously and the other eye was badly infected and had very little vision; this now became entirely destroyed. Gradually the leproma, which had involved both skin and subcutaneous tissue over large areas of the body, disappeared leaving only scars; fresh inflammatory areas ceased to appear and the patient, though blind and crippled, appeared to have either discharged or absorbed the entire infection within a period of betwen two and three years. His health recovered, his appetite was good, and he slept well.

I cannot recommend massive treatment with dyes. Former reference has been made to similar results from prolonged treatment with mercurochrome ${ }^{4}$. Smaller doses do not appear to have a curative effect and doses sufficient to cause evacuation of leproma are apt to be extremely dangerous. It would seem as if extremely concentrated dye in leproma acts as an irritant foreign body and results in its own evacuation, the bacilli and the remainder of the leproma sharing in the process. The action of dyes given in small doses over a short period to control lepra reaction is a different matter: used in this way they are safe and of considerable value.

It has been stated by Cannon and his collaborators ${ }^{5}$ that a small dose of blocking material stimulates the reticulo-endothelial system, whereas a large one interferes with its function. This might explain the difference between the actions of small and large doses of dyes in leprosy.

I have described the above case in some detail because of the resemblance that this process of sloughing out the disease bears to what sometimes occurs in neural leprosy with severely reacting major tuberculoid lesions. In these also the acute process results in phagocytosing bacilli and sloughing out diseased tissue until the granuloma is entirely absorbed or evacuated. Tuberculoid lesions are as a rule more superficial, the bacilli are few and the internal organs, if affected at all, are involved only to a negligible degree; when they flare up, though some pain and inconvenience 
is caused, varying with the extent of the lesions and the severity of the reaction, the condition is not a dangerous one. In the above described lepromatous case the patient would on several occasions have died had it not been for particularly careful medical and nursing attention. The difference between the two conditions lies in (a) the extent of the cutaneous and subcutaneous lesions, (b) the degree of the affection of internal organs, (c) the massiveness of the infection.

We are able with available treatment to control neural leprosy if diagnosis is made in time and the patient is willing to cooperate. What is most required is a means of activating the cells in early lepromatous cases before the disease has progressed to the stage of the patient described above, in which treatment with chaulmoogra and other remedies is seldom successful. Attempts at immunization in lepromatous cases by injection of lepra bacillary emulsion have been found useless; indeed the multiplication of lepra bacilli in the tissues has an inactivating rather than an immunizing effect.

If specific immunization cannot be obtained, is there nothing of the nature of group immunization? In this connection the recent experiments made with diphtheria toxoid are of interest ${ }^{6}$. And even more suggestive are the experiments of Wells and Brooke with the acid fast vole bacillus?

\section{Summary.}

I. The curative process in leprosy depends on activation of tissue cells, and the breaking down of the triple defence of the bacilli.

2. The condition produced by administration of large doses of dyes in advanced lepromatous cases is compared with that in activated major tuberculoid lesions.

3. What is most required in leprosy treatment is a means of activating the tissue cells in early lepromatous cases.

\section{REFERENCES.}

т. Pathologie et Thérapeutique de la Lepre, by P. G. Unna. Lepra VI, 3, p. I4I-I68, I906.

2. Leprosy Review, XI, 2, p. 99, April, I940.

3. Leprosy, by Rogers \& Muir, pp. 228, 240, 1940 Edition.

4. Further Notes on Mercurochrome, by E. Muir \& S. P. Chatterjee, Leprosy in India, V, I, Jan., 1933. Reprinted in Leprosy Review, IV, 3, July, I933.

5. P. R. Cannon, R. B. Baer, F. L. Sullivan \& J. R. Webster, Jl. Immunol, XVII, p. 44I, 1939.

6. Leprosy Review, XI, 3, p. I40, July, I940. Reprinted from Thai Science Bulletin II, 2, April, I940.

7. Brit. Jl. Exp. Path., XXI, 2, p. I04, Ap., I940. 